\title{
Qualidade de Vida e Perfil Nutricional de Pacientes com Câncer Colorretal Colostomizados
}

\author{
Life Quality and Nutritional Profile of Colostomized \\ Colorectal Cancer Patients.
}

\author{
RAQUEL COZERATTOLINI ${ }^{1}$; CARIN WEIRICH GALLON ${ }^{2}$
}

\begin{abstract}
${ }^{1}$ Graduanda do curso de Nutrição da Universidade de Caxias do Sul/RS; ${ }^{2}$ Nutricionista Mestre em Ciências Médicas pela Universidade Federal do Rio Grande do Sul e docente do curso de Nutrição e curso de Medicina da Universidade de Caxias do Sul/RS.
\end{abstract}

\begin{abstract}
ATTOLINI RC; GALLON CW. Qualidade de Vida e Perfil Nutricional de Pacientes com Câncer Colorretal Colostomizados. Rev bras Coloproct, 2010;30(3): 289-298.

RESUMO: Introdução: $O$ câncer colorretal, uma das neoplasias malignas mais frequentes no ocidente, é considerado a quarta causa mais comum de câncer no mundo. Objetivo: $O$ estudo teve como objetivo relacionar a qualidade de vida e o perfil nutricional em pacientes com câncer colorretal colostomizados. Metodologia: Os pacientes com idade entre 31 e 70 anos, atendidos no Ambulatório Central da Universidade de Caxias do Sul-RS, foram avaliados através do Índice de Massa Corporal (IMC), Percentual de Perda de Peso (\%PP) e Prega Cutânea Tricipital (PCT), e responderam a questões sobre fatores biológicos, familiares, sócioeconômicos, de saúde e atividade física, e para avaliação da qualidade de vida, foi utilizada a escala WHOQOL-bref. Resultados: Observou-se que a média de idade foi de 57,9 anos, $55 \%$ dos pacientes eram do sexo feminino e quanto ao IMC $65 \%$ eram eutróficos. Quanto às relações sociais, foi o domínio que apresentou pior qualidade de vida, com 63,8\%. Tratando-se da correlação IMC x domínio físico, também foi observado pior qualidade de vida (p=0,014). Conclusão: Os resultados sugerem a necessidade de realizar educação nutricional, com o intuito de orientar os pacientes na melhora da qualidade de vida, relativo aos hábitos alimentares e as relações sociais.
\end{abstract}

Descritores: Qualidade de vida, estado nutricional, colostomia, câncer colorretal, estilo de vida.

\section{INTRODUÇÃO}

Câncer é o nome dado a um conjunto de mais de 100 doenças que têm em comum o crescimento desordenado (maligno) de células que invadem os tecidos e órgãos, podendo espalhar-se (metástase) para outras regiões do corpo. ${ }^{1}$

A incidência do câncer cresce no Brasil, como em todo o mundo, num ritmo que acompanha o envelhecimento populacional decorrente do aumento da expectativa de vida. É um resultado direto das grandes transformações globais das últimas décadas, que alteraram a situação de saúde dos povos pela urbanização acelerada, novos modos de vida, novos padrões de consumo. O SUS registrou 423 mil internações por neoplasias malignas em 2005, além de 1,6 milhões de consultas ambulatoriais em oncologia. Mensalmente, são tratados cerca de 128 mil pacientes em quimioterapia e 98 mil em radioterapia ambulatorial. ${ }^{2}$

O câncer colorretal abrange tumores que atingem o cólon (intestino grosso) e o reto. Tanto homens como mulheres são igualmente afetados, sendo uma doença tratável e frequentemente curável quando localizada no intestino (sem extensão para outros órgãos). Os principais fatores de risco são: idade acima de 50 anos; história familiar de câncer de cólon e reto; histó- 
ria pessoal pregressa de câncer de ovário, endométrio ou mama; dieta com alto conteúdo de gordura, carne e baixo teor de cálcio; obesidade e sedentarismo. ${ }^{3}$ Mais de $90 \%$ dos cânceres de cólon e reto ocorrem em indivíduos com idade superior a 50 anos, e $75 \%$ atingem indivíduos sem outros fatores de risco além da idade. $^{5}$

O câncer colorretal, uma das neoplasias malignas mais frequentes no ocidente, é considerado a quarta causa mais comum de câncer no mundo e a terceira causa de incidência e mortalidade em ambos os sexos nos Estados Unidos. No Brasil, ele representa a quarta causa de óbitos, sendo que cerca de metade dos pacientes morre em menos de cinco anos, após o tratamento. O Instituto Nacional de Câncer estimou para 2010, no Brasil, 13310 casos novos de câncer colorretal no sexo masculino (14 casos novos a cada 100 mil homens) e 14800 casos no sexo feminino (15 casos novos a cada 100 mil mulheres. ${ }^{4}$

A ligação entre dieta e câncer está bem estabelecida, e estima-se que fatores de nutrição e estilo de vida sejam determinantes em um terço de todos os casos de câncer. ${ }^{7}$ A otimização da nutrição por meio do uso de alimentos específicos e seus componentes bioativos, seja individualmente, seja como parte de uma dieta controlada, é uma estratégia admissível nãoinvasiva de redução de risco. ${ }^{8}$

A colostomia é o resultado de uma cirurgia radical realizada no tratamento das patologias do trato gastrointestinal. Mais especificamente é uma derivação intestinal feita cirurgicamente na porção do intestino grosso e intestino delgado respectivamente, com fixação da alça no abdômen. Sua indicação ocorre na maioria dos pacientes atendidos pela especialidade de Colo-Proctologia em consequência de patologias crônicas como doença de Chagas, doença de Crohn, câncer, dentre outras. ${ }^{6}$

A presença do câncer altera, indubitavelmente, todos os aspectos da vida do indivíduo e pode acarretar profundas alterações no modo de viver habitual, conforme o comprometimento da capacidade e habilidade para execução de atividades de rotina. A literatura mostra que os indivíduos ostomizados enfrentam várias perdas que podem ser reais ou simbólicas. A perda do controle da eliminação de fezes e gases, condição mandatória para a vida em sociedade, pode acarretar o isolamento psicológico e social, baseado em sentimentos negativos que permeiam as relações interpessoais. ${ }^{9}$
Considerando-se as dificuldades enfrentadas pelos sobreviventes de câncer, sendo ostomizados ou não, ao submergir numa ou duas situações crônicas, câncer e ostomia, que certamente proporcionam drásticas mudanças no estilo de vida, sobrepõe-se, como foco central deste estudo, questionar como esse processo influencia a qualidade de suas vidas. ${ }^{9}$

Portanto o objetivo do estudo é relacionar a qualidade de vida com o perfil nutricional de pacientes com câncer colorretal colostomizados, podendo oferecer uma contribuição para futuros estudos e consequentemente proporcionar melhores condições de vida para os indivíduos colostomizados e não somente os com câncer colorretal.

\section{MÉTODOS}

Trata-se de um estudo transversal, em que a população de estudo foi constituída por pacientes no pós-operatório de câncer colorretal com uso de colostomia atendidos no ambulatório de oncoproctologia do Ambulatório Central da Universidade de Caxias do Sul, com idade entre 31 e 70 anos, no período de março à maio de 2010 . O serviço tem cadastrado atualmente 28 pacientes colostomizados. Foram avaliados todos os sujeitos de pesquisa que se enquadraram nos critérios de inclusão, sendo um total de 20 pacientes. Foram excluídos 8 pacientes com idade acima de 70 anos.

Para a avaliação antropométrica foi utilizado o Índice de Massa Corporal (IMC), que é um indicador simples de estado nutricional calculado a partir da seguinte fórmula: peso atual $(\mathrm{kg}) /$ estatura $\left(\mathrm{m}^{2}\right)$, interpretado segundo os valores de referência da OMS/ $1995^{10}$ para adultos e Lipschitz, D.A./ $1994^{10}$ para idosos. O peso é a soma de todos os componentes corpóreos e reflete o equilíbrio protéico-energético do indivíduo $^{11}$ e foi mensurado através de balança mecânica da marca Filizola, com estadiômetro acoplado, fornecida pelo local. A estatura foi medida utilizandose o estadiômetro ou antropômetro. Os indivíduos ficaram em pé, vestindo roupas leves, descalços, na posição ortostática e com os pés juntos ${ }^{11}$. O percentual de perda de peso (\%PP) foi obtido da relação entre o peso atual e o peso usual do paciente, refletindo a velocidade das alterações na composição corpórea e correlacionando-se com índices de morbidade e mortalidade. Também foi realizada a prega cutânea tricipital (PCT) que foi medida na face posterior do 
braço, paralelamente ao eixo longitudinal, no ponto que compreende a metade da distância entre a borda súpero-lateral do acrômio e o olecrano. Para aferição da PCT, utilizou-se a marca do ponto médio do braço direito, feita para aferição da circunferência do braço (CB), deixou-se o braço estendido, relaxado e ligeiramente afastado do corpo, segurando-se o adipômetro com uma mão e com a outra segurando a prega na região posterior do braço $\mathrm{o}^{12}$. O percentil de dobra cutânea para adultos e a circunferência do braço é classificado conforme Frisancho, $1990^{13}$.

Foi feito também uma entrevista com questões sobre fatores biológicos, no qual incluíam questões como hábito intestinal e mudança no apetite, familiares, onde o histórico familiar de doenças pregressas era questionado, socioeconômicos, que objetivou obter dados sobre a renda mensal do indivíduo, de saúde, questionando os medicamentos usados regularmente pelo entrevistado, se fumava ou ingeria bebida alcoólica e de atividade física, questionando se realizava exercício físico regularmente e com que frequencia, e dados antropométricos, como peso, altura e IMC.

Quanto à alimentação foi questionado quantas refeições o indivíduo realizava diariamente, se consumia frituras, se mudou a alimentação depois do uso da bolsa de colostomia e se tinha receio em consumir alguns tipos de alimentos; se sim, quais e porquê.

Para avaliação da qualidade de vida, foi utilizada a escala WHOQOL-bref, validada para o português no ano de 1998, composta por 26 itens que representaram as facetas, as quais, por sua vez, referiam-se a 4 domínios: físico, onde visava obter dados que avaliassem a relação do indivíduo com o seu corpo e sua percepção relativa à sua aparência física, o domínio psicológico, que buscava investigar os estados afetivos e ideativos do indivíduo, o domínio relações sociais, que objetivou levantar dados relativos ao modo como o indivíduo circula e interage no contexto familiar e social e o domínio meio ambiente, que se direcionava à análise dos fatores externos, que interferiam na vida do indivíduo, assim como a percepção deste com relação ao meio em que vive. O paciente deu seu parecer relativo a cada sintoma podendo ser classificado por escala de intensidade. A pontuação dos escores foi realizada utilizando- se o programa estatístico SPSS, com a sintaxe do WHOQOL-bref.

Os dados coletados foram tabulados em planilha Excel, 2007 e analisados através de tabelas, gráficos, estatísticas descritivas e pelos testes estatís- ticos: análise de correlação de Pearson e Teste t-student para amostras independentes.

Os resultados foram considerados significativos a um nível de significância máximo de 5\% (p<0,05) e o software utilizado para esta análise foi o SPSS versão 10,0 .

Os pacientes foram convidados a participar do estudo e assinaram o "Termo de Consentimento Livre e Esclarecido". O projeto de pesquisa foi aprovado pelo Comitê de Ética em Pesquisa da Fundação Universidade de Caxias do Sul/RS, sob o parecer 278/ 09 e está sob o cumprimento dos princípios éticos contidos na Declaração de Helsinki (2000) e conforme Resolução 196/96 do CNS (Conselho Nacional de Saúde).

\section{RESULTADOS}

Após dois meses de coleta, 20 pacientes com câncer colorretal com uso de bolsa de colostomia foram avaliados, e no que se referiu à avaliação das variáveis sexo, idade, profissão, escolaridade e estado civil, tem-se distribuição no Gráfico 1:

Em relação às variáveis quantitativas, para $\mathrm{n}=20$, observou-se que a média de idade obtida foi de 57,9 anos. Quando analisado renda, a média foi de 758,9 reais por mês. Já para escolaridade a média foi de 5,2 anos de estudo, com relação às refeições realizadas diariamente a média ficou em 4,4 refeições/dia. A média de ingestão de água por dia ficou em 1160,0 ml. Com relação à média do tempo de uso da bolsa de colostomia obteve-se um resultado de 15,1 meses ( $\mathrm{DP}=18,7)$, com tempo mínimo de 1 mês e máximo de 72 meses. O IMC teve uma média de $24,6 \mathrm{~kg} / \mathrm{m}^{2}$

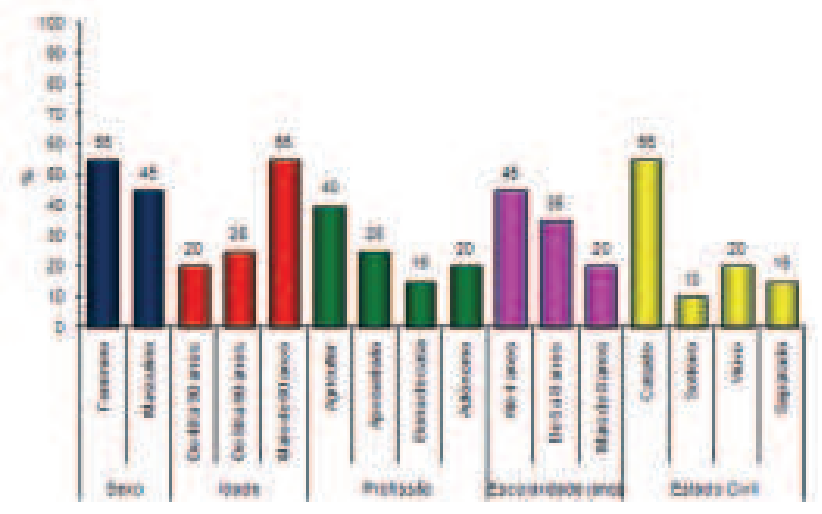

Gráfico 1 - Distribuição relativa conforme: Sexo, Idade, Profissão, Escolaridade e Estado Civil ( $n=20$ casos). 
$(\mathrm{DP}=3,4)$, no qual $65 \%$ dos casos $(\mathrm{n}=13)$ classificaram-se como eutróficos, $15 \%$ dos casos $(n=3)$ classificaram-se como desnutridos, $15 \%$ dos casos $(n=3)$ classificaram-se como sobrepeso e o restante, $5 \%$ dos casos $(n=1)$ classificaram-se como obesidade grau II. A média do percentual de perda de peso foi de 13,9, o mínimo 1,3 e o máximo 29,4. Todos os pacientes foram classificados em relação à $\mathrm{CB}$ entre baixa adiposidade e eutrofia, enquanto que para PCT a classificação ficou entre baixa adiposidade e muito alta adiposidade, referindo-se ao mínimo e ao máximo encontrados.

Observou-se a prevalência de câncer na família em $45 \%$ dos casos, e em relação a medicamentos, $40 \%$ dos sujeitos de pesquisa fazem uso de medicamento anti-hipertensivo.

Em relação à variável atividade física, não era praticada em $90 \%$ dos casos $(n=18)$. Do total da amostra, $85 \%$ dos casos $(n=17)$ não eram tabagistas, e $15 \%$ dos casos $(n=3)$ eram tabagistas, sendo que destes o número de cigarros fumados por dia variou entre $2 \mathrm{e}$ 15.

Verificou-se que $95 \%$ dos casos $(n=19)$, não consumiam bebida alcoólica. Para o hábito intestinal, teve prevalência de hábito intestinal normal para $80 \%$ dos casos $(n=16)$, hábito intestinal diarréico $15 \%$ dos casos $(n=3)$ e hábito intestinal constipado $5 \%$ $(n=1)$.

Referente às refeições realizadas diariamente, observou-se que o mínimo de refeições feitas pelos pacientes da amostra foi de 1 refeição por dia (5\% dos casos) e o máximo foi de 6 refeições por dia (10\% dos casos), sendo que $45 \%$ dos casos $(n=9)$ realizavam 5 refeições por dia. Tratando-se das frituras, $70 \%$ dos entrevistados $(n=14)$ consumiam frituras.

Após o uso da bolsa de colostomia, observouse que $45 \%$ dos casos $(n=9)$, tiveram mudança no apetite. Com relação à mudança na alimentação, 60\% dos casos $(n=12)$ relataram alteração. Para mudança na consistência dos alimentos, $85 \%$ dos casos $(n=17)$ não tiveram mudança. Sobre mudança no volume, $60 \%$ dos casos $(n=12)$ não alteraram. Em $45 \%$ dos pacientes, ocorreu mudança no tipo de alimento, destes $45 \%$ observou-se que $44,4 \%$ dos casos $(n=4)$ não ingeriam mais carne, seguido de $22,2 \%$ dos casos $(n=2)$ que não ingeriam mais feijão. Os demais pacientes não ingeriam mais saladas cruas/cozidas, alimentos fritos e leite e derivados. Considerando-se o motivo pela mudança no tipo de alimento, verificamos que 55,6\% dos casos $(n=5)$ mudaram tipo porque fazia mal, $22 \%$ dos casos $(n=2)$ mudaram porque causava gases, $11,1 \%$ mudaram porque endurecia as fezes e $11,1 \%$ dos casos $(n=1) \mathrm{mu}-$ daram porque causava diarréia.

Com relação ao receio em consumir alguns alimentos, $70 \%$ dos casos, respondeu que sim, tinham receio, destes, o maior receio foi em relação aos alimentos flatulentos que apareceu em $64,3 \%$ dos casos, seguido de carne com $35,7 \%$ e saladas cruas e leite com $28,6 \%$ cada. O motivo pelo qual os pacientes tem receio em consumir estes alimentos é na maioria dos casos $(57,1 \%)$ por produzir gases, seguido de $21,4 \%$ por causar diarréia.

Considerando o que o paciente faz quando tem intercorrências intestinais, observa-se os resultados na tabela: 1

No que diz respeito aos domínios da qualidade de vida, o que foi mais prevalente na amostra foram as relações sociais, onde $63,8 \%(\mathrm{DP}=15,6)$ dos pacientes tiveram a maior média, ou seja, as relações sociais são o domínio em que os pacientes atribuíram pior qualidade de vida, conforme analisado no Gráfico 2:

Através dos resultados da Análise de Correlação de Pearson verificou-se que existe correlação significativa apenas na correlação IMC X Domínio Físico. Observou-se que quanto maior o IMC maior tende a ser o escore da qualidade de vida para o Domínio Físico, o que revela pior qualidade de vida $(\mathrm{p}=0,014)$. As demais correlações não se mostraram significativas em relação à qualidade de vida. Conforme tabela 2 .

A partir dos resultados do teste t-student para amostras independentes verifica-se que não existe diferença significativa para os domínios da qualidade de vida entre os sexos masculino e feminino, conforme tabela 3 .

Através dos resultados do teste t-student para amostras independentes verifica-se que não existe diferença significativa para o tempo de uso da bolsa de colostomia entre os indivíduos que tiveram ou não mudança na alimentação ( $\mathrm{p}=0,411)$, entre os indivíduos que tiveram ou não mudança no apetite $(\mathrm{p}=0,129)$ e também para os que tiveram ou não receio em consumir alimentos $(\mathrm{p}=0,184)$.

\section{DISCUSSÃO}

Do ponto de vista sociodemográfico, no presente estudo, houve predomínio do sexo feminino (55\%), 
Tabela 1 - Distribuição relativa e absoluta para as variáveis relacionadas à intercorrências intestinais.

\begin{tabular}{llr}
\hline Variável e Categoria & n & \% \\
\hline O que faz quando está com diarréia & & 5 \\
$\quad$ Toma água & 1 & 25 \\
Come menos & 5 & 25 \\
$\quad$ Não come frutas & 5 & 25 \\
Toma chá & 5 & 20 \\
$\quad$ Não faz nada & 4 & 10 \\
O que faz quando está constipado & 2 & 15 \\
$\quad$ Ingere alimentos com fibras & 3 & 20 \\
Come mamão & 4 & 10 \\
Toma chá & 2 & 45 \\
Toma água & 9 & 25 \\
$\quad$ Não faz nada & & 25 \\
O que faz quando está com flatulência & 5 & 5 \\
$\quad$ Toma chá & 5 & 10 \\
Não ingere alimentos flatulentos & 1 & 35 \\
$\quad$ Reduz leite & 2 & \\
Não toma refrigerante & 7 & \\
$\quad$ Não faz nada & & \\
\hline
\end{tabular}

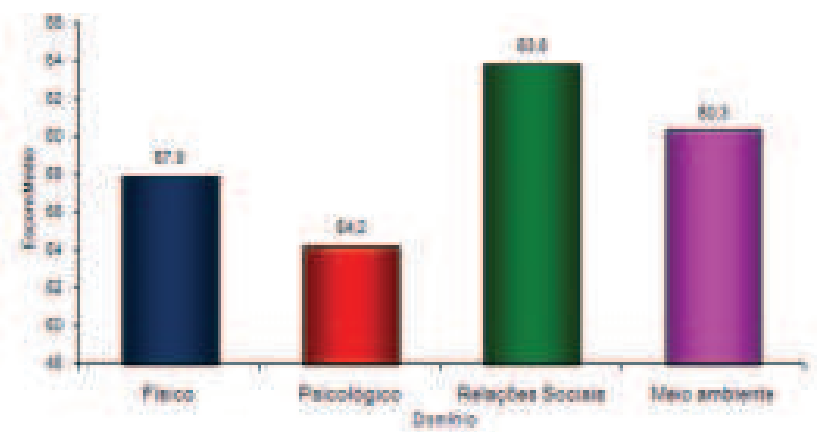

Gráfico 2 - Escore médio dos domínios da Qualidade de Vida Whoqol.

o que contradiz autores que afirmam ser o câncer colorretal mais frequente em homens ${ }^{14}$. Porém dados mais recentes do INCA estimou para 2010, no Brasil, 15 casos novos a cada 100 mil mulheres de câncer colorretal, enquanto que para homens, 14 casos novos a cada 100 mil homens ${ }^{4}$.

Com relação à idade, os dados observados na literatura indicam que cerca de $70 \%$ dos portadores desse tipo de neoplasia estão na faixa etária dos 40 aos 70 anos de idade ${ }^{15}$, o que se verificou neste estudo foi $55 \%$ dos indivíduos com mais de 60 anos. Mais recentemente foi constatado que mais de $90 \%$ dos cânceres de cólon e reto ocorrem em indivíduos com idade superior a 50 anos $^{5}$.

Outro aspecto demográfico importante é a situação conjugal dos entrevistados. Estudos internacionais com pessoas portadoras de câncer colorretal apontam entre 60 e $95 \%$ de pessoas casadas $^{15}$, o que pode ser observado neste estudo foi que $55 \%$ da amostra eram casados, e alguns estudos nacionais com indivíduos ostomizados indicam que a maior parte vive com companheiros, com percentuais de 61,9 a $87,5 \%^{14}$.

O presente estudo chama a atenção para a profissão dos indivíduos, na qual $40 \%$ eram agricultores, o que convoca novos estudos, e hipóteses com relação à alimentação dessa população que, supostamente, teria fácil acessa a uma dieta rica em frutas e verduras por terem hortas e plantações próprias. Essa suposição relativa à dieta dos agricultores segue a direção oposta dos estudos que comprovam que há uma relação inversa entre a diversidade dietética através do aumento da ingestão de hortaliças e frutas e o risco de desenvolvimento de câncer colorretal, demonstrando que esses alimentos exercem um papel crucial na etiologia dessa neoplasia ${ }^{16}$. Porém, ainda não está 
Tabela 2 - Resultado das Correlações de tempo de uso de bolsa de colostomia, \%PP, PCT e IMC com os domínios que apresentaram maior significância, e percentual de perda de peso.

\begin{tabular}{lcc}
\hline Correlação & $\mathbf{R}^{*}$ & $\mathbf{p}$ \\
\hline Tempo de Uso da bolsa de colostomia & & \\
$\quad$ X Domínio Relações Sociais & $\mathrm{R}=0,301$ & 0,197 \\
$\quad$ X Percentual de Perda de Peso (\%PP) & $\mathrm{R}=0,321$ & 0,168 \\
Percentual de perda de peso (\%PP) & $\mathrm{R}=0,400$ & 0,081 \\
$\quad$ X Domínio Físico & $\mathrm{R}=0,431$ & 0,058 \\
X Domínio Relações Sociais & $\mathrm{R}=0,409$ & 0,073 \\
$\quad$ X Domínio Meio ambiente & $\mathrm{R}=-0,405$ & 0,077 \\
Prega Cutânea Triciptal (PCT) & & \\
$\quad$ X Domínio Psicológico & $\mathrm{R}=0,540$ & 0,014 \\
IMC (Kg/m²) & $\mathrm{R}=0,431$ & 0,058 \\
$\quad$ X Domínio Físico & \\
X Domínio Meio ambiente &
\end{tabular}

*R: Coeficiente de Relação.

esclarecido na literatura qual é o determinante anticarcinogênico das frutas e hortaliças, uma vez que as mesmas são fontes de vitaminas, minerais, fibras, fitoquímicos, betacaroteno, ácido linoléico conjugado, genisteína, indóis, isotiocianatos, flavonóides, dentre outras substâncias que exercem efeito protetor contra o câncer ${ }^{16-17}$.

Verificou-se que $45 \%$ dos pacientes realizavam 5 refeições por dia, se enquadrando nas recomen- dações do guia alimentar para a população brasileira ${ }^{18}$. Neste estudo constatou-se que a média de ingestão hídrica diária foi de 1160,0ml (DP=553,8), sendo inferior às quantidades recomendadas pelo guia alimentar, de no mínimo 2 litros por dia ${ }^{18}$. Já o Consenso Nacional em Nutrição Oncológica ${ }^{19}$ recomenda para paciente adulto no pós-operatório de 18 a 55 anos, 35ml/ $\mathrm{kg} / \mathrm{dia}, 55$ a 65 anos, $30 \mathrm{ml} / \mathrm{kg} / \mathrm{dia}$ e $>65$ anos, $25 \mathrm{ml} / \mathrm{kg} /$ dia.

Tabela 3 - Número de indivíduos, média e desvio padrão, de acordo com o domínio e sexo.

\begin{tabular}{lrrc}
\hline Domínio & n & \multicolumn{2}{c}{ Escore Whoqol } \\
\cline { 2 - 4 } & & Média \pm DP & p \\
\hline Físico & 11 & $58,44 \pm 8,34$ & 0,728 \\
$\quad$ Feminino & 9 & $57,14 \pm 7,99$ & \\
$\quad$ Masculino & 11 & $55,7 \pm 17,11$ & 0,638 \\
Psicológico & 9 & $52,3 \pm 13,68$ & \\
$\quad$ Feminino & & & \\
$\quad$ Masculino & 11 & $62,88 \pm 16,4$ & \\
Relações Sociais & 9 & $64,8 \pm 15,47$ & \\
$\quad$ Feminino & & & 0,791 \\
$\quad$ Masculino & 11 & $58,52 \pm 17,5$ & 0,728 \\
Meio ambiente & 9 & $62,5 \pm 14,24$ & \\
$\quad$ Feminino & & & \\
$\quad$ Masculino & &
\end{tabular}

*DP: desvio padrão. 
A água desempenha diversas funções orgânicas vitais e imprescindíveis como transporte de gases, alimentos e produtos do metabolismo celular, regulação da temperatura corpórea, dentre outras ${ }^{20}$. Apesar da recomendação de pelo menos 2 litros de água por dia, é sabido que, em determinadas condições clínicas, como idade avançada e carcinoma do trato gastrintestinal, a necessidade de água encontrase reduzida ${ }^{21}$.

Poucos são os estudos que relacionam a ingestão de água com o câncer colorretal. Dados na literatura comprovam apenas que, dependendo do tratamento utilizado para a água destinada ao consumo humano e das substâncias nela contidas, em longo prazo, poderá haver o desenvolvimento de tipos específi$\cos$ de câncer ${ }^{22}$. Vale enfatizar que uma ingestão adequada de água auxilia nos processos metabólicos orgânicos como um todo ${ }^{20}$.

Com relação à média do tempo de uso da bolsa de colostomia, obteve-se um resultado de $15,1 \mathrm{me}-$ ses ( $\mathrm{DP}=18,7)$, com tempo mínimo de 1 mês e máximo de 72 meses, o que comprometeu algumas análises devido a enorme variação do tempo de uso da bolsa, no entanto, quando relacionadas variáveis com o tempo de uso da bolsa de colostomia, os resultados não foram estatisticamente significativos. A hipótese de que o tempo do uso da bolsa poderia interferir na qualidade de vida dos pacientes não foi confirmada no presente estudo.

Os dados antropométricos indicaram prevalência de eutrofia (65\%), encontrando-se desnutrição (15\%), sobrepeso (15\%) e obesidade $(5 \%)$. Os valores encontrados no \%PP, CB e PCT podem ser relacionados com o IMC. De acordo com a literatura, esses achados são plausíveis, uma vez que não é frequente a desnutrição no câncer de cólon e reto ${ }^{23}$. Isto pode ser explicado devido ao menor comprometimento da ingestão alimentar, ausência de distúrbios nutricionais absortivos, alterações metabólicas mínimas e inexistência de fatores obstrutivos ou efeito hormonal para a caquexia por esta neoplasia ${ }^{23}$.

Estudos científicos têm demonstrado uma associação positiva entre sobrepeso, obesidade e risco de desenvolvimento de diversos tipos de câncer, como também na mortalidade por essa doença ${ }^{23}$. Acreditase que o provável mecanismo esteja relacionado à hiperinsulinemia e ao alto nível do fator de crescimento dependente de insulina (IGF-1) e proteínas que se li- gam ao IGF-1 (IGFBP), além de dietas caracterizadas pelo consumo excessivo de energia ${ }^{24}$. Há uma maior associação entre o excesso de peso e o risco de câncer colorretal em homens quando comparado com as mulheres, sugerindo que a distribuição abdominal ou central de adiposidade corpórea (tipicamente masculina) é o principal componente do aumento desse risco, uma vez que está associada fortemente com a resistência à insulina e à hiperinsulinemia ${ }^{24}$.

De acordo com DeMarini ${ }^{17}$, a dieta é a principal fonte de exposição humana para os carcinógenos/ mutágenos e anticarcinógenos/antimutágenos ambientais, sendo que alguns tipos de câncer associados à alimentação, assim como algumas intervenções dietéticas, podem reduzir os biomarcadores ou a incidência das neoplasias.

Observou-se a prevalência de câncer na família em $45 \%$ dos pacientes, que condiz com a literatura que nos mostra que o câncer colorretal talvez seja o câncer humano com maior componente familiar. A proporção estimada de cânceres colorretais atribuível a causas hereditárias varia de 5\% a 30\%. As síndromes herdadas com defeitos genéticos conhecidos são responsáveis por 1-5\% de todos os CCR. Entre 10\% e $30 \%$ dos pacientes com CCR têm história familiar de CCR, mas não pertencem a uma síndrome hereditária conhecida $^{25}$.

No presente estudo, a prática de atividade física mostrou-se ausente em $90 \%$ dos sujeitos, não se enquadrando com o guia alimentar, que recomenda que a atividade física seja exercida pelo menos 30 minutos todos os dias ${ }^{18}$.

Estudos epidemiológicos indicam que, além de uma dieta variada com elevado consumo de frutas, hortaliças e fibras, baixo consumo de alguns tipos de gordura e ingestão calórica moderada, a prática de atividade física está intimamente relacionada ao risco reduzido de diversos tipos de câncer, particularmente, colorretal $^{26}$.

Em relação ao fumo, observou-se que $15 \%$ dos entrevistados eram tabagistas, enquanto que $85 \%$, não tabagistas. Para os fumantes, foi relatado uma quantidade entre 2 e 15 cigarros por dia. Porém, não foi investigado o tempo de consumo. Estudos científicos têm relacionado a incidência de câncer colorretal com dados sobre a quantidade de cigarros/dia e o tempo de vício, indicando que tabagistas que consomem cigarros durante muitos anos possuem um risco aumentado para adenoma colorretal e, provavelmente, câncer de cólon, 
além de elevada mortalidade por essa doença, com conseqüiente redução do risco à medida que param de fumar mais cedo ${ }^{27}$.

O consumo de frituras atual esteve presente em 70\% dos pacientes, e conforme o World Cancer Research Fund e American Institute for Cancer Research sugerem haver evidências consistentes de que dietas ricas em gorduras possam aumentar o risco de câncer colorretal ${ }^{7}$. O papel das gorduras na carcinogênese pode variar de acordo com a sua origem e composição ${ }^{28}$. Acredita-se que a elevada ingestão de gordura total promove aumento na produção de ácidos biliares, que são mutagênicos e citotóxicos? ${ }^{7}$.

Foi avaliado que $40 \%$ dos sujeitos de pesquisa faziam uso de medicamentos anti-hipertensivos, este resultado sugere que novos estudos sejam feitos a partir de doenças prévias ao câncer colorretal.

Verificou-se que apenas $5 \%$ dos casos faziam uso de algum tipo de substância alcoólica o que difere de estudos que mostram que o consumo excessivo de álcool é um fator de risco para adenomas e neoplasia colorretal $^{29}$. Este resultado pode ser atribuído ao fato de questionarmos o consumo atual e não o passado, e da possibilidade de omissão de resposta.

Quanto ao hábito intestinal dos pacientes, observou-se que $80 \%$ tinham hábito intestinal normal, isto provavelmente se explica devido ao fato de serem pacientes no pós-operatório de câncer colorretal, e no presente estudo, mostrou que o intestino normaliza após a cirurgia.

Com relação à mudança na alimentação, $60 \%$ relataram evitar alimentos flatulentos por causarem desconforto abdominal (gases) e diarréia, revelando que o uso da bolsa de colostomia implica em mudanças nos hábitos alimentares. Quando há presença de constipação e flatulência, $45 \%$ e $35 \%$ respectivamente relataram não fazer nem um tipo de intervenção nutricional, o que sugere falta de orientação em relação a estes sintomas.

Servaes ${ }^{30}$ mostrou que tem sido dada maior ênfase na qualidade de vida desta população devido aos avanços tecnológicos no diagnóstico e tratamento precoce das neoplasias malignas, que têm aumentado a sobrevida dos pacientes. Dentre os domínios de qualidade de vida avaliados, o pior escore de qualidade de vida foi associado às relações sociais, que foi a mais prevalente $(63,8 \%)$, indicando baixa qualidade de vida entre os entrevistados, semelhante ao estudo de Michelone ${ }^{9}$ que encontrou média de 64,7\% nos domínios de relações sociais em pacientes colostomizados.

Não está evidenciado na literatura a correlação entre IMC e domínio físico em pacientes colostomizados, embora o presente estudo tenha encontrado que quanto maior os índices de IMC maior os escores da qualidade de vida para o domínio físico, revelando pior qualidade de vida dos pacientes. A hipótese para este resultado poderia ser o fato do sobrepeso e obesidade estarem relacionados também ao sedentarismo, maiores riscos de doenças cardiovasculares e dores articulares.

\section{CONCLUSÃO}

Embora vários estudos tenham mostrado que hábitos de vida saudáveis e estilo de vida influenciam na qualidade de vida dos pacientes com câncer colorretal com uso de bolsa de colostomia, é preciso continuar avaliando individualmente cada caso, levando em consideração o tempo de uso da bolsa de colostomia, bem como avaliar fatores socieconômicos, ambientais e de atividade física.

Apesar de alguns resultados não serem significativos no estudo, nota-se o quanto é válido e importante continuar pesquisas que promovam melhora na qualidade de vida desses pacientes para que se possa reduzir ou prevenir a piora do quadro clínico quando se tratando de câncer colorretal.

Os resultados sugerem a necessidade de realizar educação nutricional, com o intuito de orientar os pacientes na melhora da qualidade de vida, visando mudança nos hábitos alimentares.

Considerando o apontamento de que as relações sociais se apresentam como o domínio onde a piora da qualidade de vida é mais significativa entre os sujeitos analisados, faz-se importante pensar que é imprescindível a realização de programas educacionais interdisciplinares, com atuação de Nutricionistas, Médicos, Educadores Físicos, Psicólogos, entre outros profissionais da área da saúde, que visem a otimizar o trânsito destes pacientes no contexto familiar e social, para que possam continuar inseridos na comunidade de maneira a preservar a sua dignidade e cidadania. 
Rev bras Coloproct Julho/Setembro, 2010
Qualidade de Vida e Perfil Nutricional de Pacientes com

Câncer Colorretal Colostomizados

Raquel Cozer Attolini e Cols.
Vol. 30

ABSTRACT: Introduction: Colorectal cancer, one of the most frequent malignancies in the West is considered the fourth most common cause of cancer worldwide. Objective: The study had as purpose to relate the life quality and nutritional profile in colorectal cancer colostomized patients. Methodology: The patients with ages ranging from 31 to 70 , who were attended at the Central Ambulatory from Caxias do Sul University - Rio Grande do Sul, were evaluated through BMI (Body Mass Index), \% PP (percentage of weigh loss) and PCT (tricipital skinfold), and answered questions about biological, familiar, socioeconomic, health and physical activity factors, and for life quality evaluation the scale WHOQOL-bref was used. Results: It was possible to see that the average age was 57,9 years, $55 \%$ of the patients were females and with regards to BMI $65 \%$ were eutrophic. As to social relations, this was the area that showed worse life quality, with $63,8 \%$. Regarding the correlation physical BMI x physical domain, worse life quality was also observed $(p=0,014)$. Conclusion: The results suggest the need to perform nutritional education, with the purpose of advising the patients for improvement of life quality, regarding to eating habits and to social relations.

Key words: Life quality, nutritional status, colostomy, colorectal cancer, lifestyle.

\section{REFERÊNCIAS}

1. INCAa, Instituto Nacional do Câncer. O que é o câncer?Ministério da Saúde. Rio de Janeiro, 2009.

2. INCAb, Instituto Nacional do Câncer. Magnitude do câncer no Brasil. Ministério da Saúde. Rio de Janeiro, 2009. Acesso em: 22/09/2009.

3. INCAc, Instituto Nacional do Câncer. Câncer colorretal. Ministério da Saúde. Rio de Janeiro, 2009. Acesso em: 22/09/ 2009.

4. INCA. Instituto Nacional de Câncer. Estimativa 2010: Incidência de câncer no Brasil. Rio de Janeiro. INCA, 2010.

5. Mallmann ACM, et al. Rastreamento do câncer colorretal. Rev Técnico- Científica do Grupo Hospitalar Conceição. V. 16. N. 1. P. 13-15. 2003.

6. Sonobe HM, et al. A visão do colostomizado sobre o uso da bolsa de colostomia, Revista Brasileira de Cancerologia. v. 48, n. 3. P.341-348. 2002.

7. WCRF, World Cancer Research Fund, American Institute for Cancer Research. Food, nutrition and the prevention of cancer: a global perspective. Washington: American Institute for Cancer Research, 2007.

8. Waitzberg D. Dieta, Nutrição e Câncer. Ed. Atheneu, São Paulo, 2006.

9. Michelone APC, et al. Qualidade de vida de adultos com câncer colorretal com e sem ostomia. Rev Latino-am. Enfermagem. v. 12. n. 6. P. 875-83. Nov./Dez., 2004.

10. Vitolo MR. Nutrição: da gestação ao envelhecimento. Rio de Janeiro: Ed. Rubio, 2008.

11. Cuppari L. Nutrição clínica no adulto. 2. Ed. São Paulo: Manole, 2005. 474 p.

12. Durnin, JVGA, et al. Body fat essessed from total body density and its estimation from skinfold thickness: measurements on 481 men and women aged from 16 to 72 years. Br J Nutr. n. 32. P. 77-97. 1974.

13. Frisancho AR. Anthopometric Standards of the assessment of growth and nutritional status. Ann Arbor, Mich: University of Michigan Press, 1990.
14. Bandeira RCF, Wirthmann E, Costa MQ, Kimura M, Chaves EC, Santos VLCG. Qualidade de vida e coping de pessoas portadoras de estomas definitivos. [CD-ROM] In: $4^{\circ}$ Congresso Brasileiro de Estomaterapia; $1^{\circ}$ Congresso Brasileiro de Enfermagem em Dermatologia; 2001 out 22-25; São Paulo: SOBEST; 2001.

15. Ramsey SD, Andersen MR, Etzioni R, Moinpour C, Peacock S, Potosky A, et al. Quality of life in survivors of colorectal carcinoma. Cancer 2000; 88:1294-303.

16. Garófolo A, Avesani CM, Camargo KG, Barros ME, Silva SRJ, Taddei JAAC, et al. Dieta e câncer: um enfoque epidemiológico. Rev Nutr Campinas. 2004;17(4):491-505.

17. DeMarini DM. Dietary interventions of human carcinogenesis. Mutat Res. 1998;400:457-65.

18. Ministério da saúde. Guia alimentar para a população brasileira: promovendo a alimentação saudável. Ministério da Saúde, Secretaria de Atenção à Saúde, Coordenação-Geral da Política de Alimentação e Nutrição. Brasília: MS; 2006.

19. Brasil. Ministério da Saúde. Consenso nacional de nutrição oncológica. / Instituto Nacional de Câncer. - Rio de Janeiro: INCA, 2009.

20. Continente AJC, Pluvius CC, Martinez CV. Nutrición y neoplasias digestivas. Rev Bras Nutr Clin. 2002;17:53-63.

21. Waitzberg DL. Água. In: Waitzberg DL. Nutrição oral, enteral e parenteral na prática clínica. São Paulo: Atheneu; 2000:314.

22. Doyle TJ, Zheng W, Cerhan JR, Hong CP, Sellers TA, Kushi $\mathrm{LH}$, et al. The association of drinking water source and chlorination by-products with cancer incidence among postmenopausal women in Iowa: a prospective cohort study. Am J Public Health. 1997;87(7):1168-176.

23. Calle EE, Rodriquez C, Walker-Thurmond K, Thun MJ. Overweight, obesity, and mortality from cancer in a prospectively studied cohort of US adults. N Engl J Med. 2003;348(17):1625-638.

24. Halpern A, Mancini MC. Obesidade, hiperinsulinismo e câncer In: Waitzberg DL. Dieta, nutrição e câncer. São Paulo: Atheneu; 2004:734-38. 
25. World Gastroenterology Organization/International Digestive Cancer Alliance Practice Guidelines: Triagem do câncer colorretal, 2007

26. Levi F, Pasche C, Lucchini F, Vecchia CL. Dietary fibre and the risk of colorectal cancer. Eur J Cancer. 2001;37:20912096.

27. Tranah GJ, Giovannucci E, Ma J, Fuchs C, Hankinson SE, Hunter DJ. Epoxide hydrolase polymorphisms, cigarette smoking and risk of colorectal adenoma in the Nurses'Health Study and the Health Professionals Follow-up Study. Carcinogenesis. 2004;25(7):1211-218.

28. Campos FG, Waitzberg DL, Habr-Gama A. Influência da dieta na gênese do câncer colorretal. In: Waitzberg DL. Dieta, nutrição e câncer. São Paulo: Atheneu; 2004:247-52.
29. Anderson JC, Alpern Z, Sethi G, Messina CR, Martin C, Hubbard PM, et al. Prevalence and risk of colorectal neoplasia in consumers of alcohol in a screening population. Am J Gastroenterol. 2005;100(9):2049-2055.

30. Servaes P, Verhagen S, Bleijenber G. Fatigue in cancer patients during and after prevalence, correlates and interventions. Eur J Cancer 2002;38 (1): p27-43.

Endereço para correspondência:

RAQUEL COZER ATTOLINI

Avenida Júlio de Castilhos, 2700

Bairro São Pelegrino - Caxias do Sul/RS

CEP 95010-002

Celular: (54) 91913667

Email: rakecozer@yahoo.com.br 